\title{
Hundreds of Neurons in the Aplysia Abdominal Ganglion Are Active During the Gill-Withdrawal Reflex
}

\author{
Dejan Zečević, Jian-Young Wu, Lawrence B. Cohen, Jill A. London, ${ }^{a}$ Hans-Peter Höpp, and Chun Xiao Falk \\ Department of Cellular and Molecular Physiology, Yale University School of Medicine, New Haven, Connecticut 06510, \\ and Marine Biological Laboratory, Woods Hole, Massachusetts 02543
}

\begin{abstract}
A combination of optical and electrode recording methods was used to obtain an overview of the neuron activity in the Aplysia abdominal ganglion in response to a light touch to the siphon skin. Spike activity was detected in up to 150 different neurons. Habituation and sensitization of the gillwithdrawal reflex was accompanied by large changes in the number of activated neurons. It is likely that these recordings are incomplete; the actual number of activated neurons is estimated to be about 300 in the acutely sensitized preparation. While we presume that not all $\mathbf{3 0 0}$ of these neurons are involved in the gill-withdrawal reflex, the number of neurons is so large that it may be difficult to determine the role of each activated neuron with presently available experimental tools.
\end{abstract}

The neuronal basis of the Aplysia gill-withdrawal reflex has been studied extensively using microelectrode methods (Kandel et al., 1967; Kupfermann and Kandel, 1969; Kupfermann et al., 1971; Byrne et al., 1978; Hawkins et al., 1981; Byrne, 1983; Koester, 1983; Goldberg and Lukowiak, 1983; Frost et al., 1988). Hawkins et al. (1981) described 10 interneurons or interneuron groups in the gill-withdrawal reflex and noted that the circuit was "somewhat forbidding in its complexity." Subsequently, several additional interneurons have been identified (Byrne et al., 1978; Byrne, 1983; Koester, 1983; Frost et al., 1988) and Byrne (1983) suggested that activity in the interneuron II group might be important in determining the duration of the gill withdrawal. While the total number of neurons involved in the gillwithdrawal reflex is not yet known, it has been suggested that many more interneurons are yet to be found (Frost, 1987). We have used optical recording methods in an attempt to provide an overview of the action potential activity in the abdominal ganglion that occurs during gill withdrawal.

Several forms of plasticity have been demonstrated in the gillwithdrawal reflex (Carew and Sahley, 1986). The 2 forms that

\footnotetext{
Received Feb. 21, 1989; revised Apr. 10, 1989; accepted Apr. 20, 1989.

We are grateful to Vincent Castellucci, William Frost, and Eric Kandel for demonstrating the isolated siphon preparation. We thank Rina Hildesheim, Amiram Grinvald, Leslie Loew, Joe Wuskell, and Alan Waggoner for kindly providing dyes. We thank Vic Pantani for design and construction of the extracellular electrode amplifiers. David Schiminovich and Avrum Cohen assisted in the programming. Supported by a USPHS Grant from the National Institute of Neurological and Communicative Diseases and Stroke.

Correspondence should be addressed to Dejan Zečević at his present address: Institute of Biological Research, 11000 Belgrade, Yugoslavia.

a Present address: Department of BioStructure and Function, University of Connecticut Health Center, Farmington, CT.

Copyright (c) 1989 Society for Neuroscience $0270-6474 / 89 / 103681-09 \$ 02.00 / 0$
}

we have examined are short-term habituation and short-term sensitization (Pinsker et al., 1970).

Earlier experiments showed that it was possible to record the action potential activity of individual neurons in simpler invertebrate ganglia using optical methods (Salzberg et al., 1973, 1977; Grinvald et al., 1981; London et al., 1987; Shiono et al., 1988; Yagodin et al., 1988). These methods were based on the finding that the absorption of photons by certain membranebound dye molecules is dependent on the transmembrane potential (Cohen and Salzberg, 1978; Salzberg, 1983; Grinvald et al., 1988). In experiments on buccal ganglia of Navanax, which contain 200 cell bodies, London et al. (1987) estimated that the completeness of the optical recording was $>70 \%$. However, because Aplysia abdominal ganglia contain more neurons ( 1100 or 600) (Coggeshall, 1967; Cash and Carew, 1989) and are more opaque (Boyle et al., 1983), we anticipated that the optical recordings from Aplysia ganglia would be less complete.

\section{Materials and Methods}

Experiments were carried out on the isolated siphon preparation developed by Kupfermann et al. (1971) (Fig. 1). A light mechanical stimulation $(0.5-2.5 \mathrm{gm})$ was delivered to a piece of siphon skin. The siphon was connected to the abdominal ganglion via the siphon nerve, and the ganglion was in turn connected to the gill via the brachial and genital nerves. The absence of a direct peripheral connection between the siphon and gill means that the gill withdrawal must be mediated via the abdominal ganglion.

The raw data from the optical recordings consist of cell-body spike signals on photodiode outputs; further analysis is required to determine the activity of individual neurons from this data. Since the light transmitted by a relatively large neuron will fall on several neighboring photodiodes, there will be identically timed signals on such detectors. In addition, most light paths through the ganglion will pass through several neurons and thus each detector may have signals from more than one neuron. Examples of both kinds of occurrence can be seen in the top section of Figure 2, which illustrates the outputs of 7 photodiodes (schematic drawing on the left) during a gill-withdrawal reflex. Before the touch to the siphon (indicated by the large arrow), action potentials are relatively infrequent; after the stimulus there is a dramatic increase in frequency. There are 4 easily recognized spike patterns during the period before the touch. At number 1 there is an identically timed spike signal that is seen on all of the detectors. This kind of event is repeated 1 more time before the mechanical stimulus and 19 more times after the stimulus. We presume that these signals represent the action potentials of one large neuron and the activity of this cell (1) is indicated on line 1 of the bottom section. The photodetectors with large signals from cell 1 are indicated on the bottom left. Similarly, 3 more neurons (2-4) can be identified from their spike activity before the mechanical stimulus. After the stimulus, the recordings are more complex, but, by taking into account the timing, location, signal size, and signal shape, we concluded that all of the large signals in the upper section could be accounted for by action potentials in the 4 neurons (bottom section). However, because of the limited signal-to-noise ratio in the optical measurements, the 


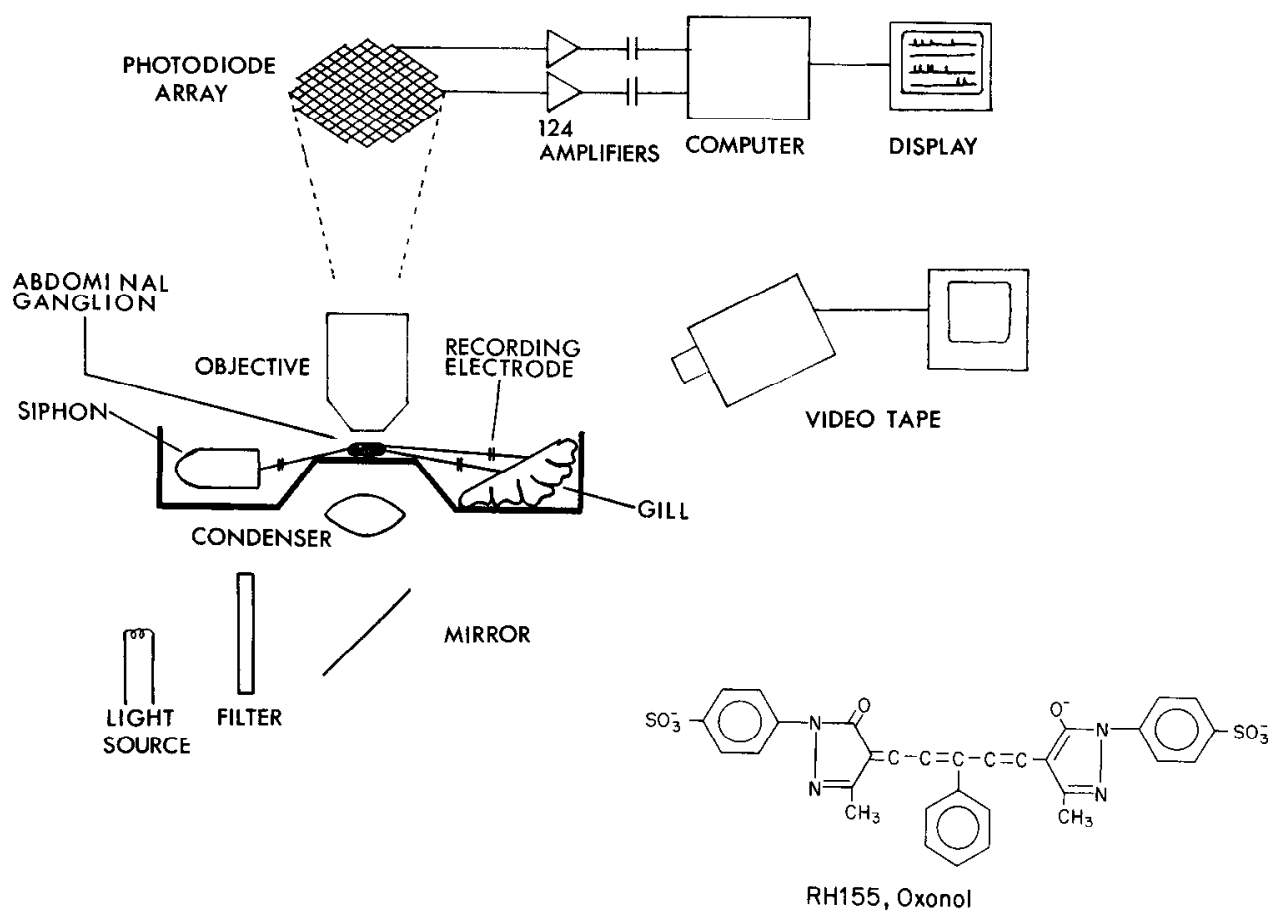

Figure 1. Schematic diagram of the apparatus. Light from a tungsten halogen lamp was passed through a $720 \pm 25 \mathrm{~nm}$ interference filter and focused on the preparation using a modification of Kohler illumination. The condenser iris was opened so that the condenser numerical aperture would equal the objective numerical aperture. We used 120 or 124 elements of a $12 \times 12$ photodiode array placed at the plane where the objective forms the real, inverted image. With this optical arrangement, the image of a 3-dimensional ganglion is formed on a 2-dimensional array. The microscope objective was focused near the middle of the ganglion with the hope of obtaining an equal signal-to-noise ratio from cell bodies on both the top and bottom surfaces of the ganglion. Since we have no information about the position of the neurons in the $Z$ axis (optical path), no attempt was made to determine if any of the individual neurons whose activity was detected optically could be identified with previously studied neurons. Photodiode currents were individually amplified. The amplifier outputs were multiplexed, digitized, and stored in a computer. The gill movements were recorded simultaneously on video tape. The structure of the dye used for these experiments is shown at lower right.

analysis was often tedious, difficult, and uncertain. Certainly, neurons with small signals that are active only in the period immediately following the mechanical stimulus are likely to be missed. This will lead to an underestimation of the number of active neurons. The top section of Figure 2 also illustrates several instances (e.g., at the small arrow) of events which have the shape of action potentials but which are too small to clearly distinguish from noise.

To determine the spike times for the individual neurons for the results illustrated in Figures 3-5, we used a program which allowed the operator to choose an individual action potential on one detector as a template. Using this template, the program determined the times of similar spike signals on this delector and then allowed the operator to see the data from neighboring detectors at those times. With this information, the operator then decided which events were generated by an individual neuron. The program then erased these spike signals from the data. In this way the raw data became progressively simplified until the spike times of the neuron with the smallest acceptable signal-to-noise ratio were recorded. The smallest acceptable signal varied somewhat from operator to operator.

Optical recordings of activity from abdominal ganglia were made using 124 elements of a $12 \times 12$ array of silicon photodiodes using methods that were modifications of those previously published (Salzberg et al., 1977; Grinvald et al., 1981; Cohen and Lesher, 1986; London et al., 1987). The diode array was placed in the image plane formed by a microscope objective of 20 or $30 \times$. The ganglion was positioned with its ventral side facing the objective and with the region adjacent to the siphon nerve imaged onto the array. With the magnification we used, about one-third of the image of the ganglion will fall outside the active area of the photodiode array and any active neurons in those regions will be missed.

The output of each detector was individually amplified. RC filters in the amplifiers limited the response of the system to $1.5-160 \mathrm{~Hz}$. Extracellular recordings were made with platinum wires touching the siphon, genital, and brachial nerves and the 2 pleural-abdominal connectives. The electrode signals were amplified $100,000 \times$ (Micro Power Systems MP843 dual-FET front end) and filtered $(10-300 \mathrm{~Hz})$. The photodiode and electrode amplifier outputs were multiplexed, digitized, and stored in a Motorola VMEbus computer. Additional filtering could be carried out digitally. The data acquisition, analysis, and display programs, in 68000 assembler and Pascal, running under VERSAdos, are available from the authors.

Preliminary experiments indicated that either the pyrazolone-oxonol, RH 155 (NK3041, lower right of Fig. 1) first suggested and synthesized by Rina Hildesheim and Amiram Grinvald, and the merocyanine-rhodanine, WW525, were better than the 27 other oxonol, merocyanine, and styryl dyes we tested on Aplysia ganglia as judged by the signal-tonoise ratio. For the experiments described here, the abdominal ganglia were stained for $10-30 \mathrm{~min}$ with a $0.5 \mathrm{mg} / \mathrm{ml}$ solution of NK304l purchased from Nippon Kankoh Shikiso Kenkyusho Co. Ltd., Okayama, Japan. The oxonol dye mainly stained the cell body layer; the neuropil region was only weakly stained. As a result, axon signals were rarely detected even with signal averaging (Grinvald et al., 1981). However, staining with a $0.5 \mathrm{mg} / \mathrm{ml}$ solution of the oxonol dye often led to irreversible inhibition of the gill-withdrawal reflex; these preparations were discarded. Recent experiments (J.-Y. Wu, C. X. Falk, H.-P. Höpp, and L. B. Cohen, unpublished observations) indicated that this staining regime also blocked spontaneous activity recorded from the siphon nerve. Thus, our recordings may have reduced amounts of spontaneous activity. Additional experiments showed that RH482 (the propyl analog of RH155, resynthesized as JPW1132 by J. Wuskell and L. Loew) and the merocyanine-rhodanines, RGA89 and JPW 1124, had reduced pharmacologic effects.

The ganglia were not desheathed. The chamber temperature was kept at $15 \pm 2{ }^{\circ} \mathrm{C}$. Preliminary reports of these experiments have appeared (London et al., 1985, 1986; Zečević et al., 1985; Wu et al., 1987; Höpp et al., 1988). 
PHOTODIODE OUTPUTS
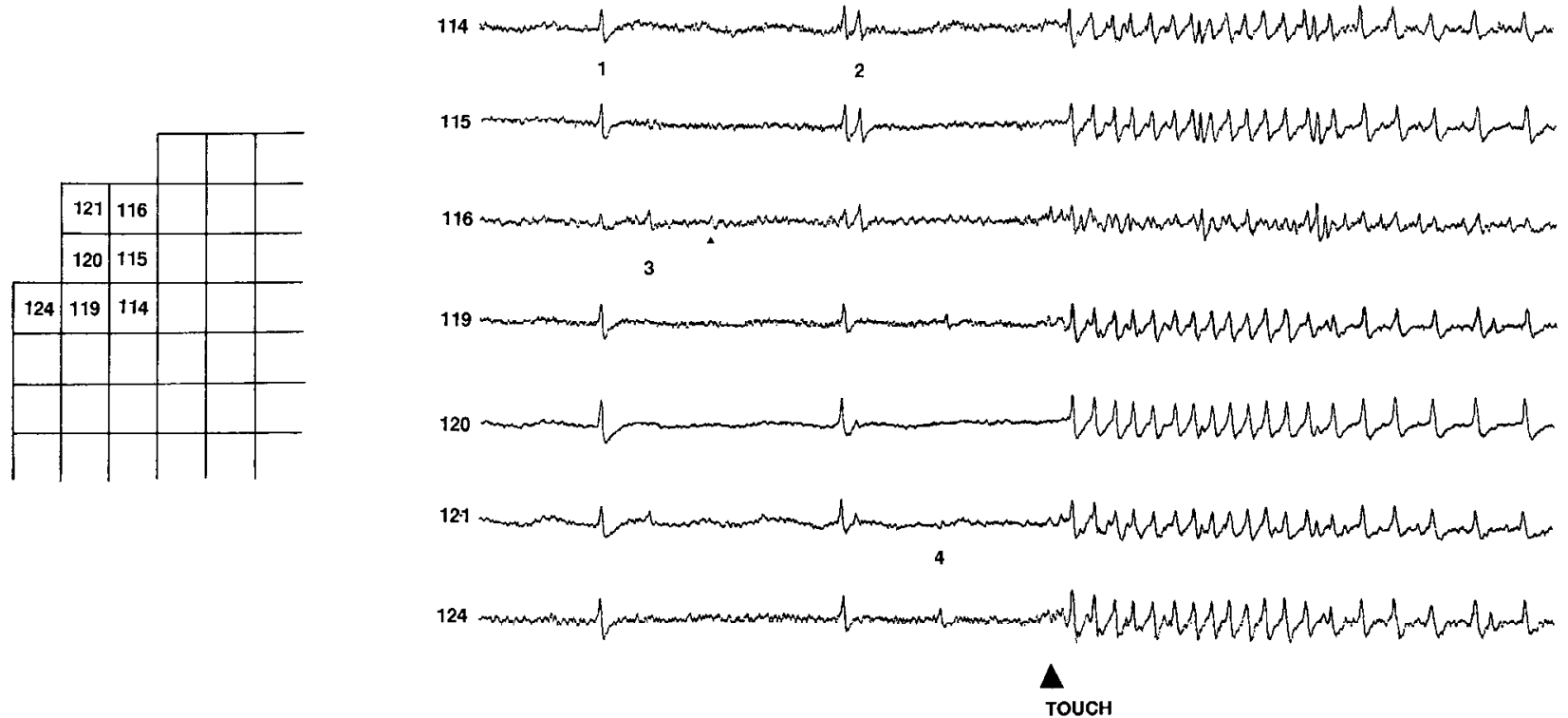

NEURON ACTIVITY
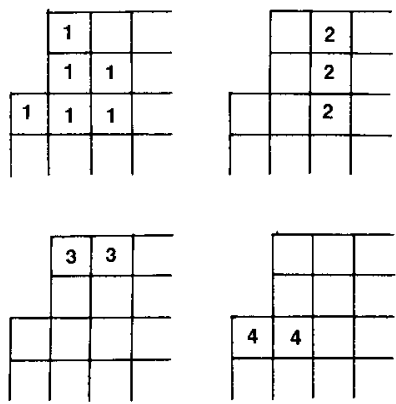

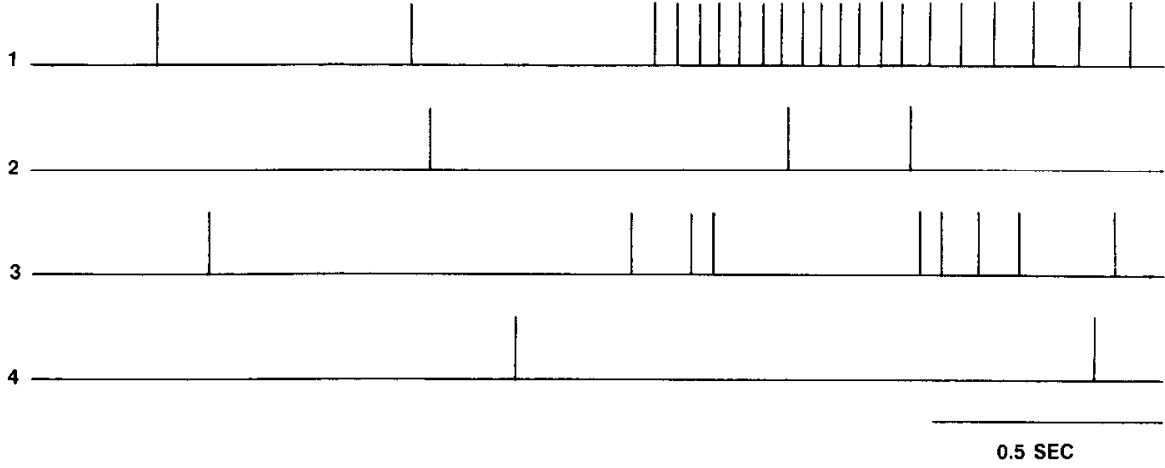

Figure 2. Comparison of photodiode outputs (top), and the analyzed results from that data, neuron activity (bottom). The outputs of 7 detectors, indicated in the drawing at left, from a portion of an $8 \mathrm{sec}$ recording are shown at the top. The numbers to the left of each trace indicate the photodetector number. Examination of the photodiode outputs indicated that activity in 4 neurons would account for all of the large optical signals. The activity of these 4 neurons is indicated in a raster diagram on the bottom. For an example of a more difficult data set and further discussion of the problems in the analysis, see figure 3 of London et al. (1987). In addition to the high-frequency filtering provided by the amplifier system, the raw data were smoothed with 2 passes of a 1-2-1 digital smoothing routine.

\section{Results}

Figure 3 illustrates the results of the analysis of the optical recordings from the 124 detectors during 3 gill withdrawals in 1 preparation. Each horizontal line represents the activity of one neuron, and each vertical tick represents one action potential in that neuron. The left section of Figure 3 shows the results obtained when the preparation was in the control state where the touch to the siphon was given less often than once in a 5 min period. In this trial, activity was detected in 103 neurons. Following this recording, 16 additional touch stimuli were delivered at a rate of $1 / \mathrm{min}$ to habituate the preparation. In the middle data set of Figure 3, the action potential activity recorded during the 16 th habituating stimulus is shown. Activity was detected in 59 neurons. Later in the experiment, a sensitizing stimulus was given to a pleuroabdominal connective. The righthand panel illustrates the activity resulting from a light touch given $10 \mathrm{~min}$ after the sensitizing stimulus. The number of active neurons was 113 , slightly more than the control, indicating that the decline in number of active neurons as a result of habituation was not simply due to deterioration of the preparation.

There did appear to be a decline in the number of active neurons with repeated recordings. In 2 preparations, the number of active neurons declined by $5-10 \%$ per recording when repeated measurements were made in the same behavioral state. This reduction in detected activity could be due to deterioration of the preparation, dye bleaching, or photodynamic damage with this dye (NK3041). If such a decline also occurred in the experiment shown in Figure 3, then the number of active neurons recorded in the sensitization trial was smaller than it would have been in the absence of this effect.

For the experiment presented in Figure 3, the mechanical stimulus was delivered by hand, and although we used a nylon bristle that was bent similarly in the 3 trials, it seems certain that the stimulus delivery was not identical from trial to trial. In the experiments illustrated in Figures 4 and 5 , the stimulus 
A. CONTROL
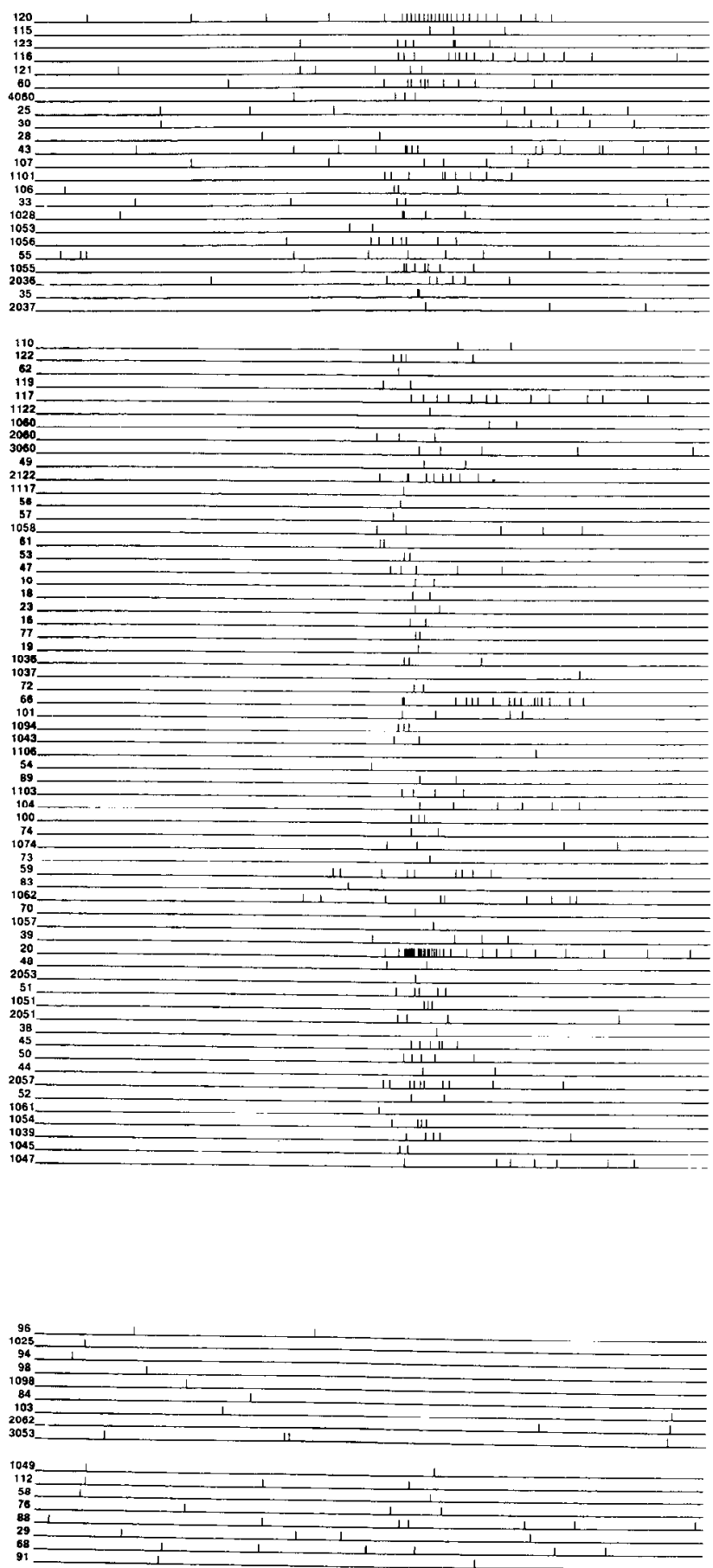

Figure 3. The action potential activity recorded optically during 3 gill withdrawals in 1 preparation. The number of active neurons was larger in the control state $(A)$ than in the habituated state $(B)$. The gill-withdrawal rcflcx was completely habituated (no movement detected) in this trail. Following sensitizing stimulation $(10 / \mathrm{sec}$ for $6 \mathrm{sec}, 1 \mathrm{msec}$ in duration) to a pleuroabdominal connective, the number of active neurons during the withdrawal $(C)$ was somewhat larger than in the control preparation. The sensitizing stimulation caused very large gill contractions and inking. The line labeled STIM at the bottom represents the approximate time of the hand-delivered mechanical stimulus. The neurons are arbitrarily arranged into 4 groups: at the top are neurons that
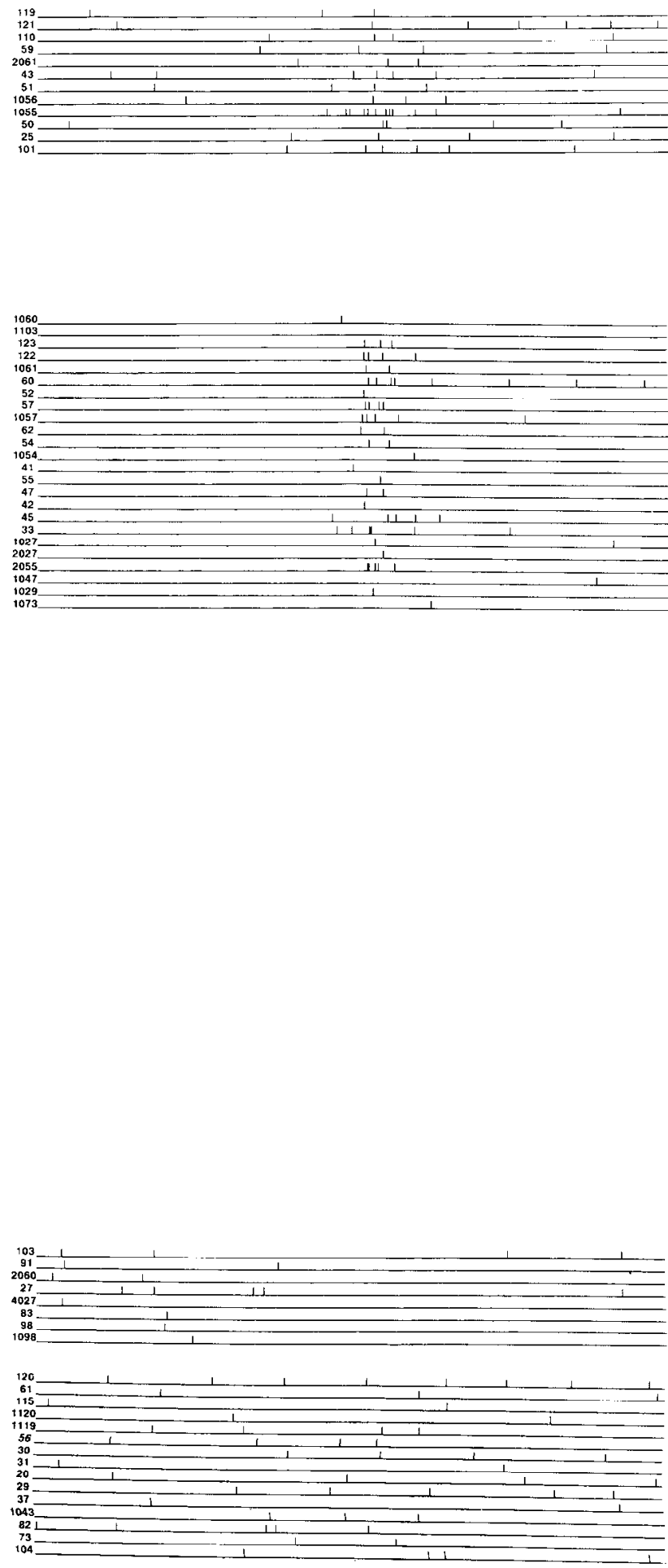

STIM

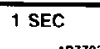

were spontaneously active before the stimulus and increased their spike frequency as a result of the stimulus; neurons which were silent before the stimulus and were activated by the stimulus make up the second group; cells whose activity was apparently decreased by the stimulus constitute the third group; and cells whose activity seemed unaffected by the stimulus comprise the fourth group. The approximate timing of the hand-delivered mechanical stimulus is indicated by the bar at the bottom. The control measurement was the first optical recording, the 
c.
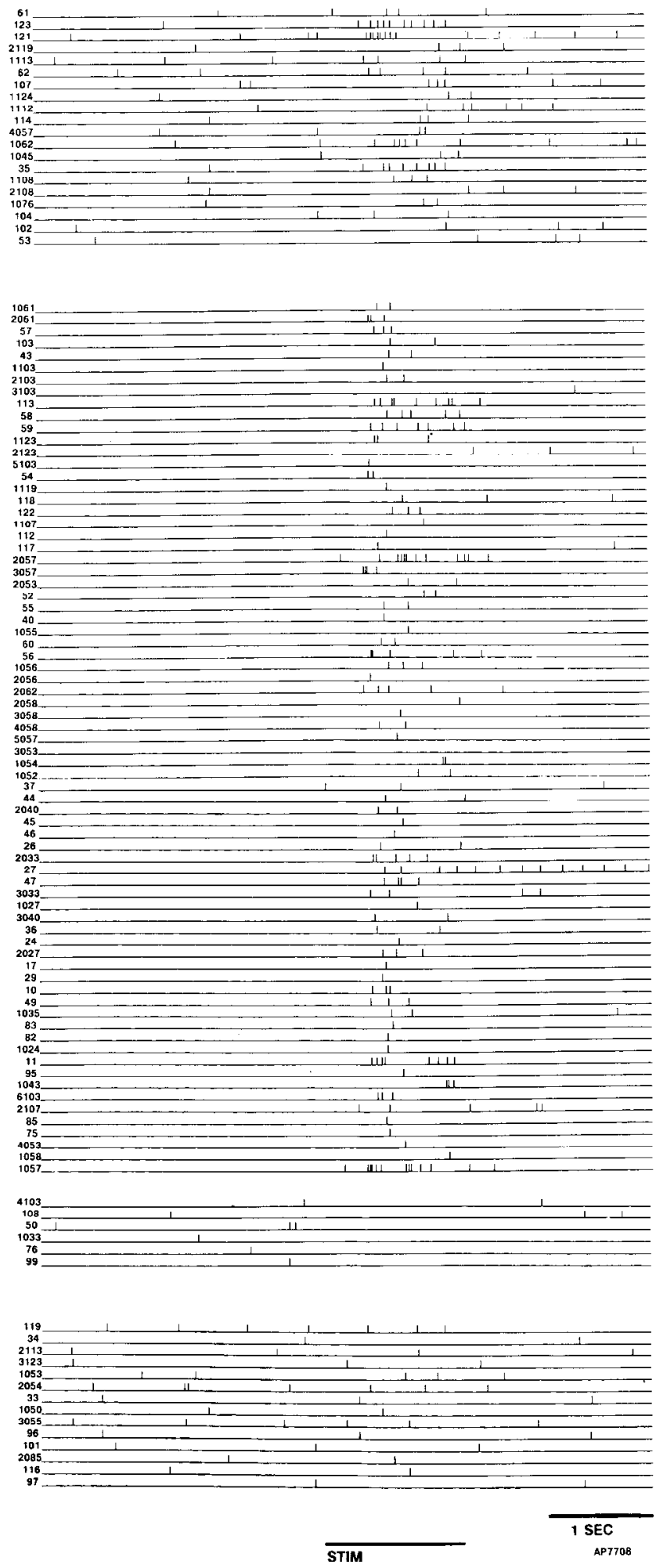

habituated measurement the third recording, and the sensitization measurement was the seventh recording in the experiment. The numbers to the left of the traces in this figure and in Figure 4 are identification numbers indicating the detector that was used for entering the spike times into the computer. Each trial in Figures 3 and 4 was analyzed independently; therefore, the identification numbers cannot be reliably used to follow neurons from trial to trial. was delivered by a computer-controlled motor at the beginning of the third second of the recording. In Figure 4 the results of 2 trials are illustrated, on the left the 12 th habituation trial and on the right a trial given $10 \mathrm{~min}$ after a sensitizing stimulation. For this experiment we also made extracellular electrode recordings from 3 nerves and 2 connectives. The activity recorded with the electrodes is indicated by the vertical black lines on the left. Activity was detected in 92 neurons ( 57 optically) in the habituated state and in 150 neurons (103 optically) in the sensitized state.

Figures 3 and 4 illustrate results from preparations which had a relatively large number of active neurons. In 6 additional preparations, the number of neurons detected optically during the gill withdrawal was between 50 and 105 in the control state. However, in other preparations, the number of optically detected neurons was smaller. Because of factors which could cause the number of detected neurons to be artifactually reduced (incomplete staining, excess optical noise, preparation in poor condition), our prejudice is that the larger numbers are more representative of the normal neuron activity.

From the results presented in Figures 3 and 4 it appears that habituation and sensitization have a large effect on the number of neurons whose activity is elicited by the touch to the siphon skin. To determine if there was also a change in the firing frequency of the neurons, we determined the number of action potentials per active neuron in each second of the optical recordings. Figure 5 illustrates 2 sets of comparisons. Figure $5 \mathrm{~A}$ compares average values from control and habituated states, and Figure $5 B$ compares average values from sensitized and habituated states. The number of neurons that were active during each second is shown on the left, and the number of spikes per second per neuron is shown on the right. The main effects of habituation and sensitization are on the number of activated neurons, although there also seems to be a small effect on the spikes per second per neuron.

The timing of action potentials detected optically was sometimes highly correlated with the timing of action potentials detected with extraccllular elcctrodes on nerves or connectives. Figure 6 illustrates 3 comparisons where each optical spike was associated with a spike on the extracellular electrode recording. We looked for similar correlations between optical and electrode recordings in 114 instances ( 3 preparations) where a cell made 3 or more action potentials that were not in the third second (where the electrode recordings were very cluttered). We found strong correlations in 21 instances. In the converse analysis, we looked to see if neuron activity identified from extracellular nerve recordings was correlated with activity recorded optically from the ganglion and found a similar fraction of strong correlations.

Because of the abdominal ganglion's relatively high opacity and large number of neurons, the recordings in Figures 3 and 4 were likely to be incomplete. The following method was used to estimate how many neurons were actually activated by the touch to the siphon. The number of neurons detected optically during a touch to the siphon in a sensitized preparation was determined. We then attempted to activate all of the neurons in the ganglion by sequentially giving electrical shocks to each nerve and connective and again determined the number of neurons whose action potential activity could be detected optically. Dividing the first number by the second gives the fraction of detectable neurons that are active during the gill withdrawal. (This assumes that the number detected during the maximal 

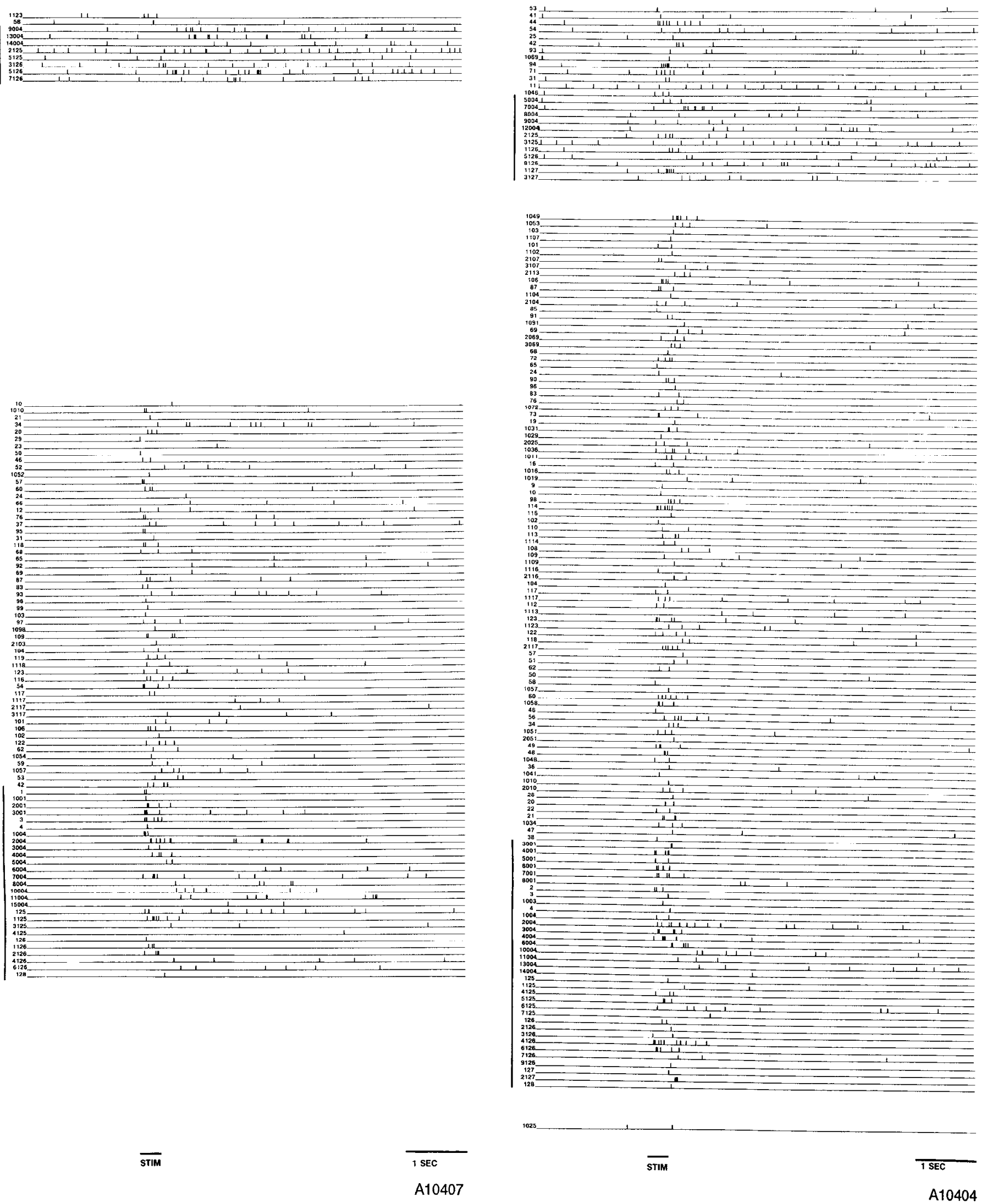

Figure 4. The action potential activity recorded optically and electrically during 2 gill withdrawals in one preparation. The number of active neurons was 150 in the sensitized state (right) and 92 in the habituated

state (left). The action potential activity in the siphon, brachial, and genital nerves and the pleuroabdominal connectives were recorded with extracellular electrodes (vertical black lines at left). When the activity 
stimulation actually represents the total number of optically detectable neurons.) In 5 preparations, this fraction was $35 \pm$ $6 \%$ (mean \pm SEM). Of the neurons whose activity was detected optically, $35 \%$ were activated by the touch to the siphon in sensitized preparations. Since the number of cell bodies present in ganglia of the size we used is either 1100 or 600 (Coggeshall, 1967; Cash and Carew, 1989), the finding that $35 \%$ of the detectablc neurons are active suggests that the actual number of active neurons during the gill-withdrawal reflex is between 200 and 400 , substantially more neurons than illustrated in either Figure 3 or 4 .

\section{Discussion}

A large fraction of the neurons which are activated by the siphon stimulus spike one or more times during the first second following the beginning of the stimulus. (A few neurons had activity which persisted for the duration of the recording.) Because of this extensive overlap in firing times, not many inferences can be made about function from the temporal sequence of spike activity in the Aplysia recordings. This was not the case in recordings made during feeding in Navanax (London et al., 1987), where neurons could be grouped according to the timing of their activity relative to the feeding behavior.

Because of the limited signal-to-noise ratio in the optical recordings, there are uncertainties in the analysis that led to the results presented in Figures 3-5. Analysis of data from 2 sequential gill withdrawals with the ganglion in the same behavioral state was carried out in 38 instances. The average difference from the mean was $18 \%$. Some of this difference presumably results from uncertainties in the analysis or operator differences and some from trial-to-trial biological variability. The analysis of the results of the maximal stimulation trials have additional uncertainties resulting from the large number of active neurons and stimulus artifacts. Thus, the estimation that the actual number of active neurons is between 200 and 400 is only an approximation. Improvements in the signal-to-noise ratio and spatial resolution will be needed to determine the number more accurately.

The conclusion that several hundreds of neurons are active during the gill-withdrawal reflex suggests that it may be very difficult to obtain an understanding of the cellular basis of this behavior. It is not easy to think of practical experiments that can answer basic questions about the circuit. For example, the demonstration that many neurons are activated by the touch to the siphon does not show that they are actually involved in the gill withdrawal. Figure 7 illustrates 2 possible neuronal organizations. Both imply that a large number of neurons would be activated by touching the siphon. In Figure $7 A$, on the left, a "labeled-line" neuronal organization is illustrated. Here, the majority of the neurons activated by the touch to the siphon are not directly involved with the gill-withdrawal reflex but instead with other aspects of the defensive withdrawal or in entirely different behaviors. However, because the gill move-

\section{$\leftarrow$}

of a neuron was detected on both optical and electrode recordings (see Fig. 6), only the result from the optical recording is shown. In this experiment, the sensitizing stimulation was given while the ganglion was habituated. Thus, the stimulation would more properly be called dishabituating. The mechanical stimulus was delivered by a computercontrolled motor. The timing of the mechanical stimulus is indicated by the bar at the bottom.
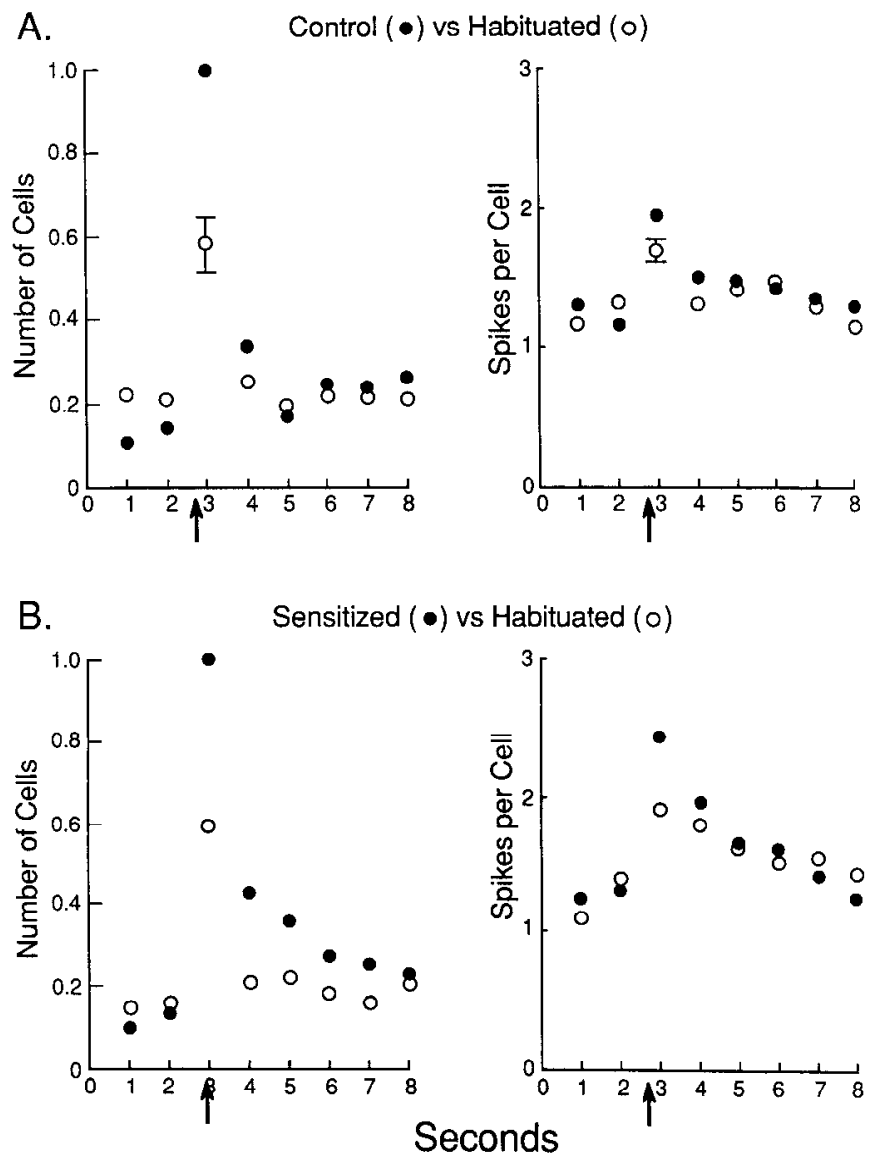

Figure 5. Comparison of the number of active neurons (left) and the number of spikes per second (right) in control, habituated, and sensitized preparations. The main effect of habituation and sensitization is on the number of active neurons, although there also appears to be a small effect on the number of spikes per cell. The touch to the siphon was delivered at the beginning of the third second (arrows). Only results from optical recordings are included. For the plots of the number of active neurons (left), the results for the control and sensitized states in the third second were normalized to 1.0 for each preparation, and this normalization factor was used for the remaining values from that preparation. $A$, Average of results from 3 preparations. In 1 preparation, measurements in the control state bracketed the measurements in the habituated state; in the other 2 preparations, the control trials came just before the habituated trials. The actual mean number of active cells in the control state for the third second was 45 . The error bar, shown for the habituated state in the third second, represents \pm the largest SEM of the non-normalized values. The spikes per cell per second are shown at right. The error bar represents \pm the average SEM for all the values. $B$, Averaged results from 2 preparations are included. In both, measurements in the habituated state bracketed the measurements in the sensitized state. The actual mean number of active cells in the sensitized state for the third second was 67 . The preparation whose results are illustrated in Figure 4 was 1 of the 2 preparations used for the comparison in $B$. The gill withdrawals (recorded on video tape) were scored by visual examination on a scale of 0 (no movement) to 5 (the largest movement to a light mechanical touch to the siphon in all of the experiments). The average for the control trials in $A$ was $1.9 \pm 0.5$; the average for the habituation trials in $A$ was $0.4 \pm 0.2$. The average for the sensitization trials in $B$ was $2.8 \pm 0.6$; the average for the habituation trials in $B$ was $0.5 \pm 0.3$.

ments are coordinated with a variety of other behaviors, this labeled-line circuit cannot be totally realistic (Altman and Kien, 1987), and a schematic drawing showing interaction among interneuron pools to provide this coordination is shown in Figure $7 B$ (middle). This view implies that some fraction of the inter- 
Figure 6. Comparison of optical signals with extracellular recordings from nerves. In the 3 instances shown, the spikes detected optically were accompanied by spikes of nearly uniform size and shape in the electrode recording. The spike signals on the optical recordings were analyzed and the spike times stored. These spike times were then used by the display program to find the identical times in the electrode data. In each comparison, the top trace is the averaged optical recording (from the cell body) and the lower traces are individual instances of extracellular electrode recordings. Results from 2 preparations are shown.

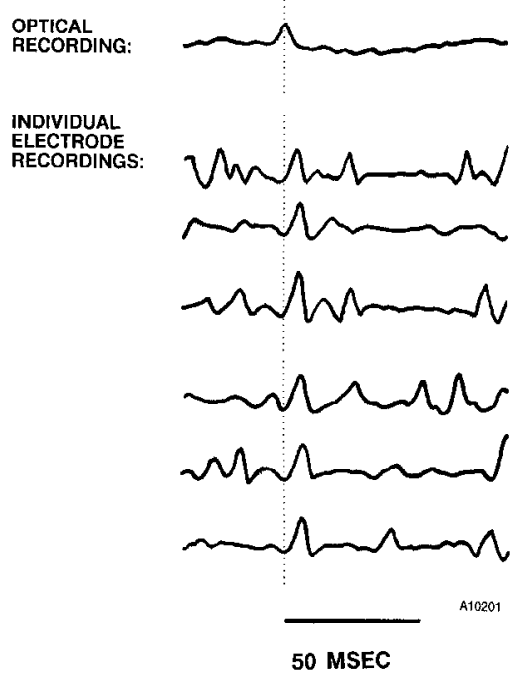

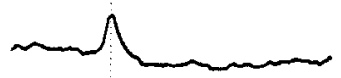

neurons in the abdominal ganglion might be involved in controlling the gill withdrawal. A more specified version is illustrated in Figure $7 \mathrm{C}$. This view considers the results from the lobster stomatogastric ganglion, where all possible pairs of interneurons have been examined by pairwise intracellular recordings (Mulloney and Selverston, 1974; Miller and Selverston, 1982), and from C. elegans, where the complete synaptic anatomy is known (White et al., 1986). In both examples there are extensive synaptic interactions between neurons. In Figure $7 C$ (on the right), 50 interneurons are shown and $25 \%$ of the pairs have synaptic interactions. If this were a realistic representation of the amount of synaptic interaction among Aplysia intcrncurons, then a relatively large fraction of the interneurons in the ganglion would be involved in the generating the gill-withdrawal reflex.

Because of the large number of neurons activated by the touch to the siphon, it may be difficult to determine which type of neuronal organization illustrated in Figure 7 is most representative. The use of cell killing and microelectrode recording methods would require identification and determination of synaptic interactions among 300 neurons; since there are about 45,000 pairs of cells to be examined for synaptic interactions, this is a formidable task. It would seem to be necessary to have a method that would allow one to quickly determine all of the synaptic followers of an impaled neuron. While it has been possible to record synaptic potentials with optical methods (Salzberg et al.,

\section{A. LABELED LINE}

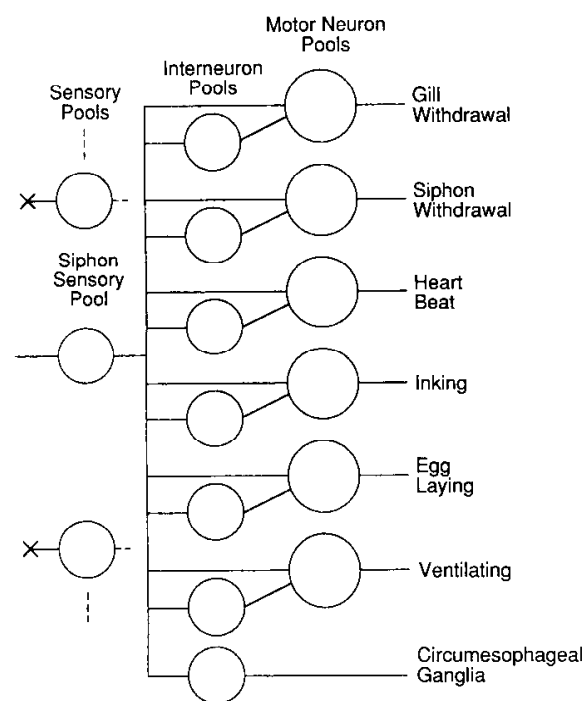

\section{B. ALTMAN-KIEN}

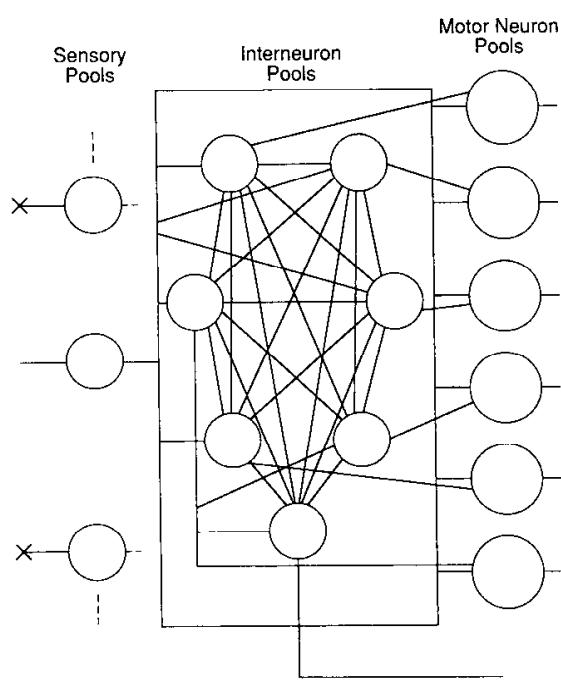

C. SCALE-UP OF STOMATOGASTRIC GANGLION

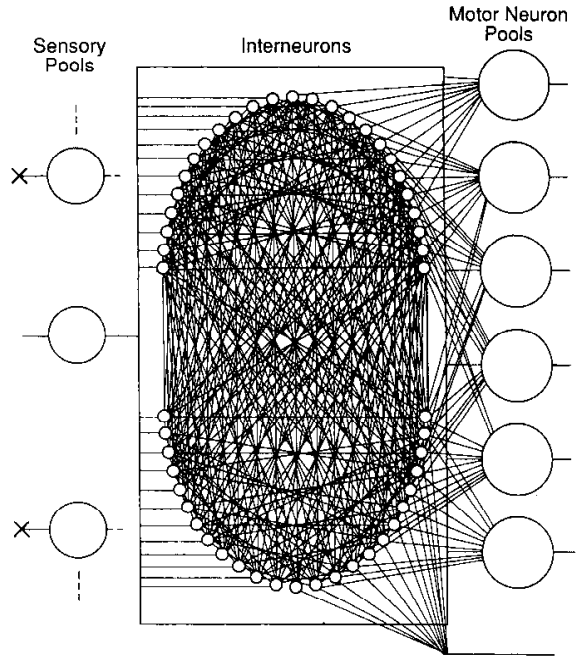

Figure 7. Schematic diagram of possible organizations of interneurons in the Aplysia abdominal ganglion. All are consistent with the finding that several hundred neurons in the ganglion are activated by a light touch to the siphon skin. In all 3 panels, the only sensory inputs indicated are from mechanosensory cells with endings in the siphon skin; the monosynaptic connections between these sensory neurons and motor neurons are also shown. $A$, "labeled line" organization. Only a few of the interneurons in the ganglion are involved in the gill-withdrawal reflex. $B$ and $C$, Interactions among interneuron pools to accomplish coordination between gill withdrawals and other behaviors controlled by the ganglion. In $C$, 50 interneurons are shown, each of which makes synaptic contact with one-quarter of the other interneurons. About 300 synaptic interactions are shown. 
1977; Grinvald et al., 1981), the signal-to-noise ratios in Aplysia ganglia are not presently great enough to allow a substantial fraction of the postsynaptic neurons to be identified. Thus, it may be very difficult to understand circuits of this complexity with presently available neurobiological tools if one means by understanding an accounting for each action potential in terms of a complete map of synaptic interactions and a complete list of the biophysical properties of each neuron.

The results presented in Figure 5 suggest that the main effects of habituation and sensitization are on the number of neurons that respond to the siphon touch rather than on the spike frequency of the activated neurons. This result is consistent with the suggestion of Byrne (1983) that interneurons may have an important role in determining plasticity. However, the results presented in Figure $5 B$ may be misleading because the firing frequencies from all activated neurons are lumped together. It would be interesting to determine the effect of habituation and sensitization on spike frequency for the subset of neurons that was active in both trials. Unfortunately, programs that would allow a comparison of neurons across trials have not yet been written.

We plan to investigate the possibility of obtaining gill withdrawals with a simplificd ncuronal circuit. Initiating the reflex by intracellular stimulation of an individual mechanosensory neuron might result in a behavior that involved fewer neurons, or perfusion of the periphery with low-calcium sea water might not affect sensory transduction or motor output but could reduce sensory feedback from the movements themselves and thereby recruit fewer neurons.

\section{References}

Altman, J. S., and J. Kien (1987) A model for decision making in the insect nervous system. In Nervous Systems in Invertebrates, M. A. Ali, ed., pp. 621-643, Plenum, New York.

Boyle, M. B., L. B. Cohen, E. R. Macagno, and H. S. Orbach (1983) The number and size of neurons in the CNS of gastropod molluscs and their suitability for optical recording of activity. Brain Res. 266: 305-317.

Byrne, J. H. (1983) Identification and initial characterization of a cluster of command and pattern-generating neurons underlying respiratory pumping in Aplysia californica. J. Neurophysiol. 49: 491-508.

Byrne, J. H., V. F. Castellucci, and E. R. Kandel (1978) Contribution of individual mechanoreceptor sensory neurons to defensive gill-withdrawal reflex in Aplysia. J. Neurophysiol. 41: 418-431.

Carew, T. J., and C. L. Sahley (1986) Invertebrate learning and memory: From behavior to molecules. Annu. Rev. Neurosci. 9: 435-487.

Cash, D., and T. J. Carew (1989) A quantitative analysis of the development of the central nervous system in juvenile Aplysia californica. J. Neurobiol. 20: 25-47.

Coggeshall, R. E. (1967) A light and electron microscope study of the abdominal ganglion of Aplysia californica. J. Neurophysiol. 30: $1263-$ 1287.

Cohen, L. B., and S. Lesher (1986) Optical monitoring of membrane potential: Methods of multisite optical measurement. Soc. Gen. Physiol. Ser. 40: 71-99.

Cohen, L. B., and B. M. Salzberg (1978) Optical measurement of membrane potential. Rev. Physiol. Biochem. Pharmacol. 83: 35-88.

Frost, W. N. (1987) Mechanisms Contributing to Short- and LongTerm Sensitization in Aplysia. Ph.D. dissertation, Columbia University, New York.

Frost, W. N., G. A. Clark, and E. R. Kandel (1988) Parallel processing of short-term memory for sensitization in Aplysia. J. Neurobiol. 19: 297-334.

Goldberg, J. I., and K. Lukowiak (1983) Transfer of habituation between stimulation sites of the siphon withdrawal reflex in Aplysia californica. Can. J. Physiol. Pharmacol. 61: 749-755.
Grinvald, A, L. B. Cohen, S. Lesher, and M. B. Boyle (1981) Simultaneous optical monitoring of activity of many neurons in invertebrate ganglia using a 124-element photodiode array. J. Neurophysiol. 45: 829-840.

Grinvald, A., R. D. Frostig, E. Lieke, and R. Hildesheim (1988) Optical imaging of neuronal activity. Physiol. Rev. 68: 1285-1366.

Hawkins, R. D., V. F. Castellucci, and E. R. Kandel (1981) Interneurons involved in mediation and modulation of the gill-withdrawal reflex in Aplysia. I. Identification and characterization. J. Neurophysiol. 45:304-314.

Höpp, H.-P., J.-Y. Wu, C. Xiao, D. Zečević, J. A. London, and I. B. Cohen (1988) Optical measurement of neuron activity during the gill withdrawal reflex in Aplysia. Abstracts, European Neuroscience Association, 352.

Kandel, E. R., W. T. Frazier, R. Waziri, and R. E. Coggeshall (1967) Direct and common connections among identified neurons in Aplysia. J. Neurophysiol. 30: 1352-1376.

Koester, J. (1983) Respiratory pumping in Aplysia is mediated by two coupled clusters of interneurons. Soc. Neurosci. Abstr. 9: 542.

Kupfermann, I., and E. R. Kandel (1969) Neuronal controls of a behavioral response mediated by the abdominal ganglion of Aplysia. Science 164: 847-850.

Kupfermann, I., H. Pinsker, V. Castellucci, and E. R. Kandel (1971) Central and peripheral control of gill movements in Aplysia. Science 174: 1252-1256.

London, J. A., D. Zečević, and L. B. Cohen (1985) Simultaneous optical recording from many cells from Aplysia abdominal ganglia during the gill-withdrawal reflex. Soc. Neurosci. Abstr. 11: 642.

London, J. A., L. B. Cohen, and D. Zečević (1986) Simultaneous optical recording from many cells from Aplysia abdominal ganglia during the gill-withdrawal reflex. Soc. Neurosci. Abstr. 12: 397.

London, J. A., D. Zečević, and L. B. Cohen (1987) Simultaneous optical recording of activity from many neurons during feeding in Navanax. J. Neurosci. 7: 649-661.

Miller, J. P., and A. I. Selverston (1982) Mechanisms underlying pattern generation in lobster stomatogastric ganglion as determined by selective inactivation of identified neurons. IV. Network properties of the pyloric system. J. Neurophysiol. 48: 1416-1432.

Mulloney, B., and A. I. Selverston (1974) Organization of the stomatogastric ganglion of the spiney lobster. I-III. J. Comp. Physiol. 91: 178.

Pinsker, H., I. Kupfermann, V. Castellucci, and E. R. Kandel (1970) Habituation and dishabituation of the gill-withdrawal reflex in Aplysia. Science 167: 1740-1742.

Salzberg, B. M. (1983) Optical recording of electrical activity in neurons using molecular probes. In Current Methods in Cellular Neurabiology, J. L. Barker and J. F. McKelvy, eds., pp. 139-187, Wiley, New York.

Salzberg, B. M., H. V. Davila, and L. B. Cohen (1973) Optical recording of impulses in individual neurones of an invertebrate central nervous system. Nature 246: 508-509.

Salzberg, B. M., A. Grinvald, L. B. Cohen, H. V. Davila, and W. N. Ross (1977) Optical recording of neuronal activity in an invertebrate central nervous system: Simultaneous monitoring of several neurons. J. Neurophysiol. 40: 1281-1291.

Shiono, S., M. Nakashima, S. Yamada, and M. Maeda (1988) Multichannel optical recording of neural activities during Aplysia gill withdrawal reflex. Second International Symposium on Bioelectronic and Molecular Elcctronic Devices, pp. 57-60.

White, J. G., E. Southgate, J. N. Thomson, and S. Brenner (1986) The structure of the nervous system of the nematode Caenorhabditis elegans. Phil. Trans. R. Soc. London 314: 1-340.

Wu, J.-Y., D. Zečević, J. A. London, M. Rioult, and L. B. Cohen (1987) Optical measurement of neuron activity during the gill withdrawal reflex in Aplysia. Soc. Neurosci. Abstr. 13: 817.

Yagodin, S. V., V. M. Slutsky, V. L. Suhanov, Yu. L. Slonimsky, V. M. Stolovicky, V. P. Valyuhov, and S. V. Popov (1988) Spatiotemporal characteristics of potential-dependent optical signals of molluscan ganglia obtained with a 100 -element photodiode array. Dokl. Acad. Sci. USSR 298: 1497-1502.

Zečević, D., J. A. London, and L. B. Cohen (1985) Simultaneous optical recording from many cells from Aplysia abdominal ganglia using fluorescence. Neurosci. Lett. 22: 370. 